\title{
Current-Mode Biquadratic Filters Using Dual Output Current Conveyors
}

\author{
Yichuang Sun and Barry Jefferies \\ Department of Electrical and Electronic Engineering, University of Hertfordshire, \\ Hatfield AL10 9AB, U.K \\ Email: y.sun@herts.ac.uk, Tel: ++44 1707 284196, Fax: ++44 1707284199
}

\begin{abstract}
A number of novel current-mode two integrator loop active filters incorporating dual output second-generation current conveyors (DO-CCII), resistors (R) and capacitors (C) are systematically presented. These DO-CCIIRC biquadratic architectures consist of 2-4 DO-CCIIs and 2-6 resistors depending on the structure and only 2 capacitors. They offer various filter characteristics such as the LP, BP, HP and BS and have very low sensitivity. They are also suitable for integration, of low parasitic effects and tunable by grounded resistors, because all capacitors and resistors in the structures are grounded.
\end{abstract}

\section{Introduction}

Active filters using second-generation current conveyors (CCIIs) have been widely investigated [1-7]. Many individual voltage- and current-mode structures have been published. Literature search has shown that most CCIIRC filters in the literature use single output CCIIs [1-4], which makes it not very easy to produce "true" currentmode filters, because there may not be suffcient output terminals that can be used to directly output or feedback currents. It has also been observed that most currentmode CCII-RC filters take output currents from capacitor or resistor branches $[4,5]$. Such current output methods are not suitable for integration, increase the effects of nonideal CCII input and output impedances and parasitic node capacitances, and cause coupling if the driven stage does not have zero input impedance in the cascade design, since they will actually result in floating capacitors or resistors. Utilizing the current following and virtual grounding property of the CCII with $y$ terminal grounded, we may insert extra CCIIs into the associated branches to take current outputs. But the nonidealilties of these extra CCIIs will severely degrade the filter performance.

The dual output operational transconductance amplifier (DO-OTA) has been earlier used in current-mode filter design [8]. A recent study of current-mode DO-OTA$C$ filters has further shown that multiple current output devices are very useful in the generation of current-mode filter structures [9], since using these output terminals, current outputs and current feedback can be readily accomplished. This study motivates us to utilize the dual output CCII (DO-CCII) to solve the problems mentioned above. In this paper, we generate current-mode DOCCII-RC filters. It should be noted that due to the lack of systematic search, some filter structures published are found to be a simple repetition of others and some common performance features of a category of filters have been frequently repeated. Thus, rather than giving one or two filters, many current-mode DO-CCII-RC filters of the two integrator loop structure are systematically presented.

\section{Current-Mode DO-CCII-RC Filter Structures}

The basic current-mode DO-CCII-RC building block is indicated in Fig.1(a), having a transfer relationship $I_{o 1}=$ $\frac{g_{1}}{Y}\left(I_{i 1}+I_{i 2}+I_{i 3}\right)$. The ideal integrator and amplifier correspond to $Y=s C_{1}$ and $Y=g_{2}$ respectively. The current summer is realized simply with the circuit node. Firstorder current-mode filters are shown in Fig.1(b) and (c) respectively. With $\tau_{1}=C_{1} / g_{1}$ and $k_{11}=g_{2} / g_{1}$, Fig.1(b) has the LP function $I_{o 1}=I_{i 1} /\left(\tau_{1} s+k_{11}\right)$. Fig.1(c), with $\tau_{1}=C_{1} / g_{1}$ and $k_{11}=g_{2} / g_{3}$, has the LP and HP transfer functions $I_{o 1}=\left(I_{i 1}-k_{11} I_{i 2}\right) /\left(\tau_{1} s+k_{11}\right), \quad I_{o 2}=$ $\left(k_{11} I_{i 1}+k_{11} \tau_{1} s I_{i 2}\right) /\left(\tau_{1} s+k_{11}\right)$. A canonical LP structure $\left(k_{11}=1\right)$ is shown in Fig.1(d), having the transfer function $I_{o 1}=I_{i 1} /\left(\tau_{1} s+1\right)$.

Four general second-order current-mode DO-CCII-RC filters of the two integrator loop structure are shown in Fig.2. Fig.3 shows six special current-mode two integrator loop DO-CCII-RC configurations. With $\tau_{1}=C_{1} / g_{1}$ and $\tau_{2}=C_{2} / g_{2}$ and $k_{i j}$ 's as exhibited in Table 1 , the current transfer functions of Fig.2(a), (b), (c) and (d) are formulated as shown in Eqns.(1), (2), (3) and (4) respectively. The current transfer functions of Fig.3(a), (b), (c), (d), (e) and (f) reduce to Eqns.(5), (6), (7), (8), (9) and (10) respectively. 
Table 1: Parameter relations of current-mode realizations

\begin{tabular}{c|cccccc}
\hline Fig. & $2(a)$ & $2(b)$ & $2(c)$ & $2(d)$ & & \\
\hline$k_{11}$ & $\frac{g_{3}}{g_{4}}$ & & $\frac{g_{3}}{g_{1}}$ & & & \\
$k_{22}$ & & $\frac{g_{4}}{g_{5}}$ & & $\frac{g_{3}}{g_{2}}$ & & \\
$k_{12}$ & $\frac{g_{5}}{g_{6}}$ & $\frac{g_{3}}{g_{5}}$ & $\frac{g_{4}}{g_{5}}$ & $\frac{g_{4}}{g_{5}}$ & & \\
\hline & & & & & & \\
\hline Fig. & $3(a)$ & $3(b)$ & $3(c)$ & $3(d)$ & $3(e)$ & $3(f)$ \\
\hline$k_{11}$ & $\frac{g_{3}}{g_{4}}$ & 1 & $\frac{g_{3}}{g_{4}}$ & 1 & $\frac{g_{3}}{g_{1}}$ & \\
$k_{22}$ & & & & & & $\frac{g_{3}}{g_{2}}$ \\
$k_{12}$ & 1 & $\frac{g_{5}}{g_{6}}$ & $\frac{g_{3}}{g_{4}}$ & 1 & 1 & 1 \\
\hline
\end{tabular}

$D_{1}(s) I_{o 1}=\tau_{2} s I_{i 1}-k_{12} I_{i 2}+k_{11} \tau_{2} s I_{i 3}+k_{12} \tau_{2} s I_{i}$ $D_{1}(s) I_{o 2}=I_{i 1}+\left(\tau_{1} s+k_{11}\right) I_{i 2}+k_{11} I_{i 3}+k_{12} I_{i 5}$

$D_{1}(s) I_{o 3}=-k_{11} \tau_{2} s I_{i 1}+k_{11} k_{12} I_{i 2}+k_{11}\left(\tau_{1} \tau_{2} s^{2}\right.$

$\left.+k_{12}\right) I_{i 3}-k_{11} k_{12} \tau_{2} s I_{i 5}$

$D_{1}(s) I_{o 5}=-k_{12} I_{i 1}-k_{12}\left(\tau_{1} s+k_{11}\right) I_{i 2}$

$-k_{11} k_{12} I_{i 3}+\left(k_{12} \tau_{1} \tau_{2} s^{2}+k_{11} k_{12} \tau_{2} s\right) I_{i 5}$

$D_{1}(s)\left(I_{o 3}+I_{o 5}\right)=-\left(k_{11} \tau_{2} s+k_{12}\right) I_{i 1}$

$-k_{12} \tau_{1} s I_{i 2}+k_{11} \tau_{1} \tau_{2} s^{2} I_{i 3}+k_{12} \tau_{1} \tau_{2} s^{2} I_{i 5}$

$D_{2}(s) I_{o 1}=\left(\tau_{2} s+k_{22}\right) I_{i 1}-k_{12} I_{i 2}+k_{12} \tau_{2} s I_{i 34}$

$D_{2}(s) I_{o 2}=I_{i 1}+\tau_{1} s I_{i 2}+\left(k_{22} \tau_{1} s+k_{12}\right) I_{i 34}$

$D_{2}(s) I_{03}=-k_{12} I_{i 1}-k_{12} \tau_{1} s I_{i 2}+k_{12} \tau_{1} \tau_{2} s^{2} I_{i 34}$

$\left.D_{2}(s) I_{o 4}=-k_{22} I_{i 1}-k_{22} \tau_{1} s I_{i 2}+k_{22} \tau_{1} \tau_{2} s^{2} I_{i 34}\right\}$

$D_{1}(s) I_{o 1}=\tau_{2} s I_{i 1}-k_{12} I_{i 2}+k_{12} \tau_{2} s I_{i 4}$

$D_{1}(s) I_{o 2}=I_{i 1}+\left(\tau_{1} s+k_{11}\right) I_{i 2}+k_{12} I_{i 4}$

$D_{1}(s) I_{o 4}=-k_{12} I_{i 1}-\left(k_{12} \tau_{1} s+k_{11} k_{12}\right) I_{i 2}$

$+\left(k_{12} \tau_{1} \tau_{2} s^{2}+k_{11} k_{12} \tau_{2} s\right) I_{i 4}$

$D_{2}(s) I_{o 1}=\left(\tau_{2} s+k_{22}\right) I_{i 1}-k_{12} I_{i 2}$

$+k_{12}\left(\tau_{2} s+k_{22}\right) I_{i 4}$

$D_{2}(s) I_{o 2}=I_{i 1}+\tau_{1} s I_{i 2}+k_{12} I_{i 4}$

$D_{2}(s) I_{o 4}=-k_{12} I_{i 1}-k_{12} \tau_{1} s I_{i 2}+\left(k_{12} \tau_{1} \tau_{2} s^{2}\right.$

$\left.+k_{12} k_{22} \tau_{1} s\right) I_{i 4}$

$$
\left.\begin{array}{l}
D_{1}(s) I_{o 1}=\tau_{2} s I_{i 1}-I_{i 2}+k_{11} \tau_{2} s I_{i 3} \\
D_{1}(s) I_{o 2}=I_{i 1}+\left(\tau_{1} s+k_{11}\right) I_{i 2}+k_{11} I_{i 3} \\
D_{1}(s) I_{o 3}=-k_{11} \tau_{2} s I_{i 1}+k_{11} I_{i 2} \\
+k_{11}\left(\tau_{1} \tau_{2} s^{2}+1\right) I_{i 3} \\
D_{1}(s)\left(I_{o 3}-I_{o 2}\right)=-\left(k_{11} \tau_{2} s+1\right) I_{i 1} \\
-\tau_{1} s I_{i 2}+k_{11} \tau_{1} \tau_{2} s^{2} I_{i 3} \\
D_{1}(s) I_{o 1}=\tau_{2} s I_{i 1}-k_{12} I_{i 2}+k_{12} \tau_{2} s I_{i 5} \\
D_{1}(s) I_{o 2}=I_{i 1}+\left(\tau_{1} s+1\right) I_{i 2}+k_{12} I_{i 5} \\
D_{1}(s) I_{o 5}=-k_{12} I_{i 1}-k_{12}\left(\tau_{1} s+1\right) I_{i 2} \\
+k_{12}\left(\tau_{1} \tau_{2} s^{2}+\tau_{2} s\right) I_{i 5} \\
D_{1}(s) I_{o 1}=\tau_{2} s I_{i 1}-k I_{i 2}+k \tau_{2} s I_{i 3} \\
D_{1}(s) I_{o 2}=I_{i 1}+\left(\tau_{1} s+k\right) I_{i 2}+k I_{i 3} \\
D_{1}(s) I_{o 3}=-k\left(\tau_{2} s+1\right) I_{i 1}-k \tau_{1} s I_{i 2} \\
+k \tau_{1} \tau_{2} s^{2} I_{i 3}
\end{array}\right\}
$$

$$
\begin{aligned}
& D_{1}(s) I_{o 1}=\tau_{2} s I_{i 1}-I_{i 2} \\
& D_{1}(s) I_{o 2}=I_{i 1}+\left(\tau_{1} s+1\right) I_{i 2} \\
& D_{1}(s) I_{o 1}=\tau_{2} s I_{i 1}-I_{i 2} \\
& D_{1}(s) I_{o 2}=I_{i 1}+\left(\tau_{1} s+k_{11}\right) I_{i 2} \\
& D_{2}(s) I_{o 1}=\left(\tau_{2} s+k_{22}\right) I_{i 1}-I_{i 2} \\
& D_{2}(s) I_{o 2}=I_{i 1}+\tau_{1} s I_{i 2}
\end{aligned}
$$

where

$$
\begin{gathered}
D_{1}(s)=\tau_{1} \tau_{2} s^{2}+k_{11} \tau_{2} s+k_{12}, \\
\omega_{o}=\sqrt{k_{12} /\left(\tau_{1} \tau_{2}\right)}, Q=\left(1 / k_{11}\right) \sqrt{k_{12} \tau_{1} / \tau_{2}} \\
D_{2}(s)=\tau_{1} \tau_{2} s^{2}+k_{22} \tau_{1} s+k_{12}, \\
\omega_{o}=\sqrt{k_{12} /\left(\tau_{1} \tau_{2}\right)}, Q=\left(1 / k_{22}\right) \sqrt{k_{12} \tau_{2} / \tau_{1}}
\end{gathered}
$$

The parameter relations of $k_{12}, k_{11}$ and $k_{22}$ for all the general and special realizations are presented in table 1. When $k_{i j}=1$, the corresponding feedback paths reduce to simple pure wire connections. $D_{1}(s)$ or $D_{2}(s)$ of each structure can be determined by substituting the corresponding $\tau_{j}$ and $k_{i j}$ into Eqns.(11) and (12).

From the expressions in Eqn.(1) we can see that Fig.2(a) can perform LP, BP, HP and BS filtering functions. Eqn.(2) indicates that Fig.2(b) supports LP, BP, and HP filters. Eqns.(3) and (4) reveal that Fig.2(c) and (d) provide LP and BP characteristics. Eqn.(5) shows that Fig.3(a) realizes LP, BP, HP and BS filters. Eqns.(6), $(8),(9)$ and (10) indicate that Fig.3(b), (d), (e) and (f) offer the LP and BP functions. From Eqn. (7) we can see that the filter configuration in Fig. 3(c) offers the LP, BP and HP characteristics.

All the structures in Figs. 2 and 3 are constructed based on current integrators and current amplifiers. Fig.2(a) and (b) contain four DO-CCIIs; Fig.2(c), (d) and Fig.3(a), (b) and (c) all require three DO-CCIIs; Fig.3(d), (e) and (f) two. Fig.2(a) requires six resistors; Fig.2(b), (c), (d) five; Fig.3(a), (b), (c) four; Fig.3(e) and (f) three; Fig.3(d) two. All the structures in Figs.(2) and (3) contain only two capacitors. Also, all resistors and capacitors are grounded.

It is clear that $\omega_{o}, \omega_{o} / Q$ and $Q$ may be tuned by controlling the associated grounded conductances $g_{j}$ at $\mathrm{x}$ terminals. The sensitivities of $Y=\omega_{o}, \omega_{o} / Q, Q$ to $x=C_{j}$, $g_{j}\left(S_{x}^{Y}=\frac{x}{Y} \frac{\partial Y}{\partial x}\right)$ are either 0, or $\pm 1 / 2$, or \pm 1 .

Universal current-mode synthesis can be realized by adding the input current distributor for some output, or the output current summer for some input, or the input/output combiner to any basic current-mode DOCCII-RC structures generated [10]. The input current distributor and output current summer can be implemented using CCIIs. 

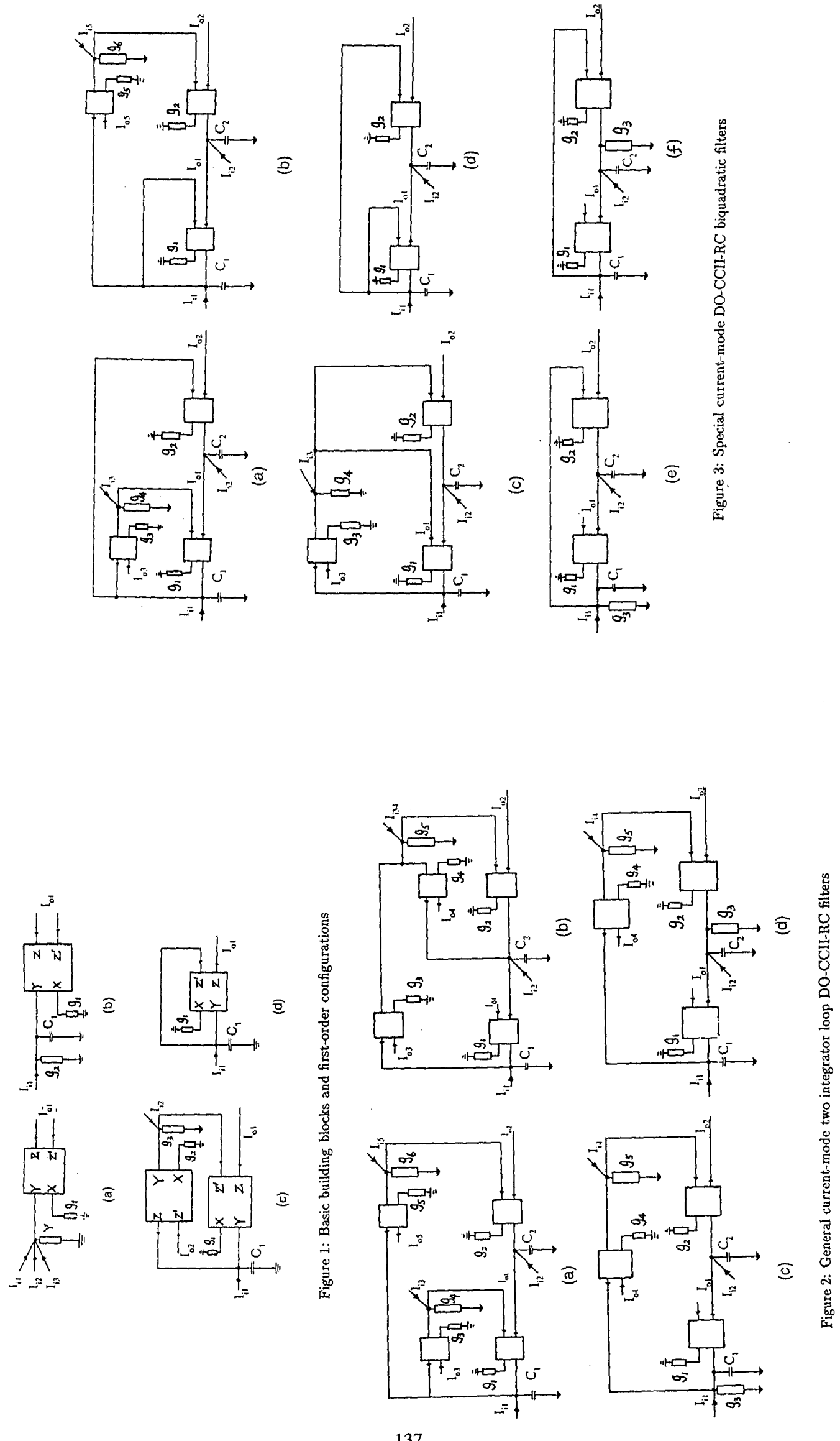


\section{Conclusions}

We have presented a variety of low sensitivity currentmode two integrator loop filter architectures using dual output CCIIs, grounded resistors and grounded capacitors. We have also shown the range of filter functions which are supported by the various configurations. The effects of the current and voltage tracking errors of the CCII can be evaluated and compensated using the methods proposed in [7]. The effects of other CCII nonidealities including the input and output impedances and frequency dependence can also be considered.

\section{References}

[1] C. Toumazou, F. Lidgey and D. G. Haigh (eds), Analogue IC design: the current-mode approach, Peter Peregrinus, London, 1990.

[2] A. S. Sedra, G. W. Roberts and F. Gohh, "The current conveyor: history, progress and new results, "IEE Proc. pt. $G$, vol.137, pp.78-87, 1990.

[3] Y. Sun anf J. K. Fidler, "Versatile active biquad based on second-generation current conveyors," Int. J. Electron., vol.76, pp.91-98, 1994 .

[4] A. Fabre and M. Alami, "Universal current mode biquad implementated from two second generation current conveyors," IEEE Trans. Circuits Syst.-I, pp.383-385, 1995.

[5] G. S. Moschytz and A. Carlosena, "A classification of current-mode single-amplifer biquads based on a voltageto-current transformation," IEEE Trans. Circuits Syst. $I I$, vol.41, pp.151-156, 1994.

[6] Y. Sun and J. K. Fidler, "Some design methods of OTA-C and CCII-RC active filters," Digest. IEE Saraga Colloq. Filters, London, 1993.

[7] Y. Sun anf J. K. Filder, "Analysis of current conveyor error effects in signal processing circuits," Int. J. Cir. Theor. Appl., vol.24, pp.479-487, 1996.

[8] J. K. Fidler, R. J. Mack and P. D. Noakes, "Active filters incorporating the voltage-to-current transactor", Microelectron. J., vol.8, pp.19-22, 1977.

[9] Y. Sun and J. K. Fidler, "Structure generation of currentmode two integrator loop dual output-OTA grounded capacitor filters," IEEE Trans. Circuits Syst., Part II, vol.43, No.9, 1996.

[10] Y. Sun and J. K. Fidler, "Design of current-mode multiple output OTA and capacitor filters," Int. J. of Electronics,vol.81, pp.95-99, 1996.

[11] Y. Sun and J. K. Fidler, "High-order current-mode continuous-time multiple output OTA capacitor filters," IEE 15th Saraga Colloq. Digital and Analogue Filters and Filtering Systems, pp.8/1-8/6, London, 1995.

[12] Y. Sun and J. K. Fjdler, "Current-mode OTA-C realjsation of arbitrary filter characteristics," Electronics Letters, vol.32, pp.1181-1182, 1996. 